\title{
Robust design optimization of a renewable-powered demand with energy storage using imprecise probabilities
}

\author{
Diederik Coppitters ${ }^{1,2^{*}}$, Ward De Paepe ${ }^{1}$, and Francesco Contino ${ }^{3}$ \\ ${ }^{1}$ University of Mons, Thermal Engineering and Combustion Unit, 7000 Mons, Belgium \\ ${ }^{2}$ Vrije Universiteit Brussel, Fluid and Thermal Dynamics, 1050 Brussels, Belgium \\ ${ }^{3}$ Université Catholique de Louvain, Institute of Mechanics, Materials and Civil Engineering, 1348 Louvain-la-Neuve, Belgium
}

\begin{abstract}
During renewable energy system design, parameters are generally fixed or characterized by a precise distribution. This leads to a representation that fails to distinguish between uncertainty related to natural variation (i.e. future, aleatory uncertainty) and uncertainty related to lack of data (i.e. present, epistemic uncertainty). Consequently, the main driver of uncertainty and effective guidelines to reduce the uncertainty remain undetermined. To assess these limitations on a grid-connected household supported by a photovoltaic-battery system, we distinguish between present and future uncertainty. Thereafter, we performed a robust design optimization and global sensitivity analysis. This paper provides the optimized designs, the main drivers of the variation in levelized cost of electricity and the effect of present uncertainty on these drivers. To reduce the levelized cost of electricity variance for an optimized photovoltaic array and optimized photovoltaic-battery design, improving the determination of the electricity price for every specific scenario is the most effective action. For the photovoltaic-battery robust design, the present uncertainty on the prediction accuracy of the electricity price should be addressed first, before the most effective action to reduce the levelized cost of electricity variance can be determined. Future work aims at the integration of a heat demand and hydrogen-based energy systems.
\end{abstract}

\section{Introduction}

PhotoVoltaic (PV) systems are the main driver of the global renewable energy capacity growth [1]. However, to reach $100 \%$ renewable electricity supply by $2050, \mathrm{PV}$ systems alone are inadequate due to solar intermittency. To address this intermittency, battery energy storage enables to store electricity and overcome the timeperiod when solar irradiance is insufficient to cover the demand [2]. Designing such PV-battery systems are subject to parameter uncertainty, such as the uncertainty on the solar irradiance, electricity demand and investment costs over the lifetime [3]. Arun et al. consider the solar irradiance stochastic in the sizing of PV-battery systems and provide the sizing curves for different levels of confidence [4]. Maleki et al. consider the solar irradiance, the wind speed and the load as stochastic and propagate these uncertainties through the model by Monte Carlo Simulation [5]. Zheng et al. consider the climate conditions and economic parameters uncertain through precise distributions and illustrate the conditions in which battery capacity is optimized in a renewable microgrid [6].
In these studies that consider uncertainty, the applications are limited to generic uncertainty characterization. Such precise distribution representation does not differentiate between uncertainty related to lack of data (i.e. present, epistemic uncertainty), and uncertainty related to the actual natural variation of the parameter (i.e. future, aleatory uncertainty). Therefore, it is unclear what measures should be taken to reduce the uncertainty efficiently on the predicted techno-economic objective. In this work, a new method is applied to characterize, propagate and evaluate parameter uncertainty in a hybrid renewable energy system framework. The method integrates over the different scenarios possible for parameters subject to variation in the future (e.g. electricity price, electricity demand) and considers the uncertainty on each scenario itself (i.e. natural variation on the outcome for each scenario). The results can be presented in a single probability box, which enables to compare design performances directly, as opposed to providing separate results for each scenario. The method is integrated in a robust design optimization approach, which aims to minimize the mean and standard deviation of the probability box upper-bound.

\footnotetext{
* Corresponding author: diederik.coppitters@umons.ac.be
} 
In section 2, the system model is described, followed by the uncertainty characterization, uncertainty quantification and robust design optimization strategies. The results in section 3 provides the set of optimized designs and their performance in Levelized Cost Of Electricity (LCOE) mean and standard deviation. Section 3 concludes with the characterization of the main drivers of the LCOE uncertainty for different optimized designs through a global sensitivity analysis. Section 4 comprises the main conclusions of the work.

\section{Method}

In this section, the photovoltaic system model and battery system model are presented. The uncertainty characterization of the parameters that define these models is discussed, followed by the uncertainty quantification method to propagate these uncertainties through the model and quantify their effect on the model output. Finally, the robust design optimization algorithm is illustrated.

\subsection{Photovoltaic-battery system model}

A grid-connected household is considered, supported by a PV-battery system (Figure 1). The PV array and battery stack are connected via a DC-DC converter to a DC bus bar. A DC-AC inverter connects the DC bus bar to the load and the electricity grid. When the PV array complies with the demand, excess PV electricity is stored in the battery. Otherwise, when the PV electricity fails to meet the demand, the battery is discharged, supported by the grid as soon as the minimum battery State Of Charge (SOC) is reached.

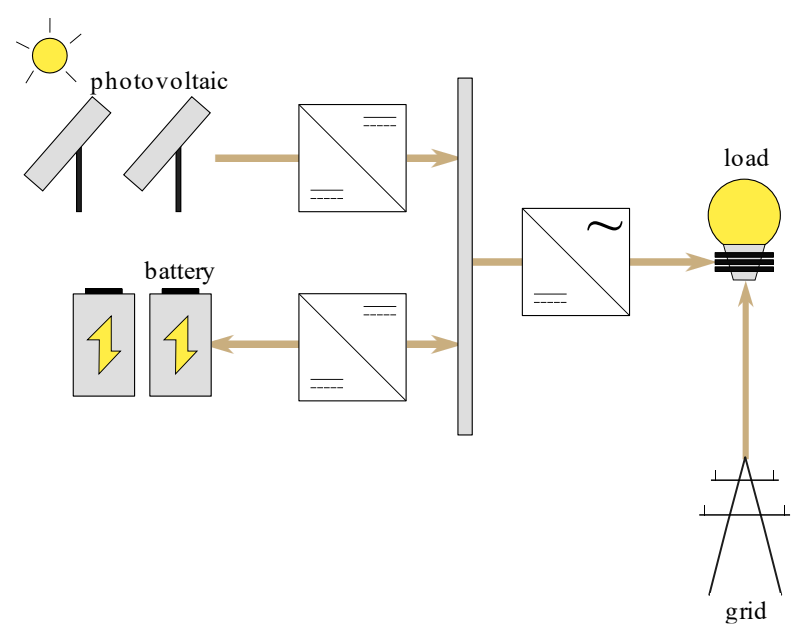

Fig. 1. Schematic of the PV-battery system

The PV array is characterized by the experimentallyvalidated model out of the PVlib Python library [7]. This model adopts the single-diode equation to characterize the current-voltage characteristic:

$$
U_{\mathrm{PV}}=I_{\mathrm{L}}-I_{0}\left\{\exp \left[\left(U+I R_{\mathrm{s}}\right) /\left(n_{\mathrm{d}} N_{\mathrm{s}} U_{\mathrm{th}}\right)\right]-1\right\}-\left(U+I R_{\mathrm{s}}\right) /\left(R_{\mathrm{sh}}\right) .
$$

The parameters in Equation 1 are characterized through the method presented by De Soto et al. [8], based on manufacturer data.

To store excess power of the PV system, we selected the lead-acid battery technology. The lead-acid battery model is adopted from Blaifi et al. [9], which quantifies the voltage-current relation as:

$$
U_{\text {bat }}=U_{\text {bat }}+I_{\text {bat }} R_{\text {bat }},
$$

where the current $I_{\text {bat }}$ is positive during charging of the battery and negative during battery discharge. The resistance $R_{\text {bat }}$ is characterized based on operating temperature, current and capacity. The battery lifetime is determined based on the hourly SOC variations over the lifetime through the Rainflow cycles counting method [10]. We refer to Blaifi et al. for the detailed quantification of the parameters in Equation 2 during charge and discharge [9].

\subsection{Climate and load data}

In this work, Brussels (Belgium) is considered as the evaluated location. To characterize the climate and the dwelling demand, a Typical Meteorological Year (yearly solar irradiance: $1118 \mathrm{kWh} /$ year, average yearly ambient temperature: $10.7^{\circ} \mathrm{C}$ ) and a synthetic dwelling demand profile for one year with hourly resolution (electricity demand: $3.98 \mathrm{MWh} /$ year) are adopted, respectively $[11,12]$. We adopted the method presented by Montero Carerro et al. to adapt the demand profile to the climate and human behaviour in Belgium [13].

\subsection{Robust design optimization}

To characterize the robust design optimization framework, first the design parameters and objective are present. Then, the uncertainty characterization and uncertainty quantification methods are illustrated, followed by the overall optimization algorithm.

\subsubsection{Design parameters and objective}

The capacity of the PV array and battery stack are selected as independent design parameters, which means that the optimization algorithm can exclude storage whenever beneficial (i.e. set the battery stack capacity to $0 \mathrm{kWh}$ ). The LCOE is selected as the technoeconomic system objective and can be determined by the annual system cost and the annual electricity demand $E_{\text {dem }}[14]$ :

$$
\mathrm{LCOE}=\left(\mathrm{CAPEX}_{\mathrm{a}}+\mathrm{OPEX}_{\mathrm{a}}+C_{\mathrm{r}, \mathrm{a}}+C_{\mathrm{e}, \mathrm{a}}\right) / E_{\mathrm{dem}} .
$$

To determine the annual system cost, the annualized investment cost $\mathrm{CAPEX}_{\mathrm{a}}$, operational cost $\mathrm{OPEX}_{\mathrm{a}}$, replacement $\operatorname{cost} C_{\mathrm{r}, \mathrm{a}}$ and the grid electricity $\operatorname{cost} C_{\mathrm{e}, \mathrm{a}}$ at retail price (wholesale price is assumed equal to $30 \%$ of the retail price) are added together. We refer to Zakeri et al. for the detailed quantification of the parameters [14].

Additionally, the Self-Sufficiency Ratio (SSR) is defined as the amount of electricity covered by the PV- 
battery system, divided by the total electricity demand. The SSR is an indicator for adopters of the HRES, as it illustrates the resilience against large electricity price increases and the protection against power cuts.

Table 1. Uncertainty characterization of the input parameters.

\begin{tabular}{|c|c|c|c|}
\hline & Type & Mean & $\begin{array}{c}\text { Standard } \\
\text { deviation }\end{array}$ \\
\hline $\begin{array}{c}\text { annual } \\
\text { electricity } \\
\text { demand }\end{array}$ & $\begin{array}{c}\text { present } \\
+ \text { future }\end{array}$ & $\begin{array}{c}{[3.61,5.22]} \\
\mathrm{MWh} / \mathrm{year}\end{array}$ & $\begin{array}{c}{[0.01,0.16]} \\
\mathrm{MWh} / \mathrm{year}\end{array}$ \\
\hline $\begin{array}{c}\text { wholesale } \\
\text { electricity price }\end{array}$ & $\begin{array}{c}\text { present } \\
+ \text { future }\end{array}$ & $\begin{array}{c}{[57,89]} \\
€ / \mathrm{MWh}\end{array}$ & $\begin{array}{c}{[0.3,4.8]} \\
€ / \mathrm{MWh}\end{array}$ \\
\hline $\begin{array}{c}\text { annual solar } \\
\text { irradiance }\end{array}$ & future & $\begin{array}{c}1118 \\
\mathrm{kWh} / \mathrm{year}\end{array}$ & $2.9 \%$ \\
\hline $\begin{array}{c}\text { yearly average } \\
\text { ambient } \\
\text { temperature }\end{array}$ & future & $10.7^{\circ} \mathrm{C}$ & $0.3^{\circ} \mathrm{C}$ \\
\hline $\begin{array}{c}\text { CAPEX } \\
\text { PV }\end{array}$ & present & $\begin{array}{c}{[430,780]} \\
€ / \mathrm{kW}\end{array}$ & \\
\hline CAPEX $_{\text {bat }}$ & present & $\begin{array}{c}{[102,352]} \\
€ / \mathrm{kWh}\end{array}$ & \\
\hline $\begin{array}{c}\text { Interest rate } \\
{[4,8] \%}\end{array}$ & present & & \\
\hline
\end{tabular}

\subsubsection{Uncertainty characterization}

The uncertainty on the model parameters are characterized by their present (epistemic) and future (aleatory) uncertainty [15]. The present uncertainty is related to lack of knowledge and can be reduced by gaining more information on the parameter (e.g. several scenarios can be constructed for the electricity price 10 years from now). The future uncertainty relates to the unknown evolution of the parameter, which is usually predicted with a certain error (e.g. the uncertainty on the electricity price for each scenario). To characterize the present and future uncertainty on a parameter, a parametric probability-box (p-box) is used [16]. Such a p-box is defined by a range on the mean $\mu$ and standard deviation $\sigma: \mu \in\left[\mu_{\min }, \mu_{\max }\right], \sigma \in\left[\sigma_{\min }, \sigma_{\max }\right]$. The truebut-unknown distribution (Gaussian), characterized by $\mu$ and $\sigma$, represents the future uncertainty of each parameter, while the range on the statistical moments represents the present uncertainty (Uniform).

The wholesale electricity price and electricity demand are characterized by a p-box. For the electricity price, 6 scenarios define the present uncertainty, i.e. base case scenario, decentral scenario and large-scale renewable energy system scenario, each determined in the coal-before-gas merit order and gas-before-coal merit order [17]. The future uncertainty comes from the prediction error for each scenario, based on the prediction errors on the electricity price reported in the retrospective review of the Annual Energy Outlook [18]. A similar philosophy is applied for the electricity demand, where 4 different scenarios represent the present uncertainty and the prediction error indicates the future uncertainty $[18,19]$. The solar irradiance and ambient temperature are characterized by a Typical
Meteorological Year (TMY), which is assumed valid over the lifetime of the system. Nevertheless, the interannual variability, determined based on historical data between 1995 - 2015, represents the future uncertainty [20]. Finally, the fixed interest rate, CAPEX of the PV array [21] and CAPEX of the battery stack [22] are subject to present uncertainty. These parameters are fixed at the beginning of the project and are therefore only subject to the current lack of confidence during system design. A summary of the uncertainty characterization is provided in Table 1 .

\subsubsection{Uncertainty quantification}

To propagate the uncertainties through the model and quantify the statistical moments of the LCOE, we considered a computationally efficient sparse Polynomial Chaos Expansion (PCE) method, which has been developed in our research group [23] and adapted to be able to propagate both precise distributions and parametric probability boxes [16]. The PCE method enables to characterize a surrogate model $M^{\mathrm{PCE}}$ of the real model $M$, based on a set of orthogonal polynomials $\boldsymbol{\Psi}$ and corresponding coefficients $u$, which are quantified through a set of real model evaluations:

$$
M^{\mathrm{PCE}}(\boldsymbol{\xi})=\sum_{i=0}^{p} u_{\mathrm{i}} \boldsymbol{\Psi}_{\mathrm{i}} \approx M(\boldsymbol{\xi}) .
$$

Once the coefficients are quantified, analytical postprocessing can be performed to determine the Sobol' indices. These indices indicate the contribution of each stochastic parameter to the variance of the objective and provides valuable information on the system behaviour under uncertainty. Additional details on this sparse PCE method are described by Abraham et al. [23]. In this work, a polynomial order of 3 is required to approximate the real model behaviour accurately $(<1 \%$ error on the statistical moments, compared to a Monte Carlo Simulation result).

\subsubsection{Robust Design Optimization}

In this RDO framework, minimizing the mean and standard deviation of the LCOE are selected as objectives [24]. While minimizing the LCOE mean is beneficial for the average expected cost of electricity paid by the system owner, reducing the LCOE standard deviation increases the probability of operating near that LCOE mean in reality. As in this specific uncertainty characterization the model output is characterized by a probability box, the mean and standard deviation of the upper probability bound of the probability box are selected, to guarantee a robust prediction on the LCOE [25]. The sparse PCE method is coupled to the Nondominated Sorting Genetic Algorithm (NSGA-II) to find the set of designs that presents the trade-off between minimizing the mean and standard deviation of the LCOE [26-28]. Hence, for every evaluated design, the sparse PCE method is applied to quantify the statistical moments. The optimization algorithm is characterized with a population of 20 samples, a crossover and mutation probability of 0.9 and 0.1 respectively. 


\section{Results}

In this section, the robust design optimization provides the set of optimized designs. The Sobol' indices are quantified for three optimized designs and guidelines are extracted from the Sobol' indices to reduce the LCOE variance of these designs.

\subsection{Robust design optimization}

The robust design optimization results illustrate the trade-off between minimizing the mean and minimizing the standard deviation of the LCOE upper probability bound (Fig. 2.). The lowest mean (318 €/MWh) is achieved by a PV array of $2.2 \mathrm{~kW}_{\mathrm{p}}$. Reducing the LCOE standard deviation is subsequently achieved by increasing the PV array capacity, followed by the inclusion of a battery stack. This subsequent increase in capacity improves the SSR, which consequently decreases the dependency on the grid electricity price uncertainty. To illustrate, the PV-design with the lowest LCOE mean achieves an SSR of $29 \%$, while the PVbattery robust design (i.e. lowest LCOE standard deviation) achieves an SSR of 58\%. The designs achieving an SSR $>58 \%$ carry an uncertainty in electricity demand and solar irradiance which overcompensates the reduction in uncertainty related to grid electricity and are therefore not considered in the Pareto set of optimized design.

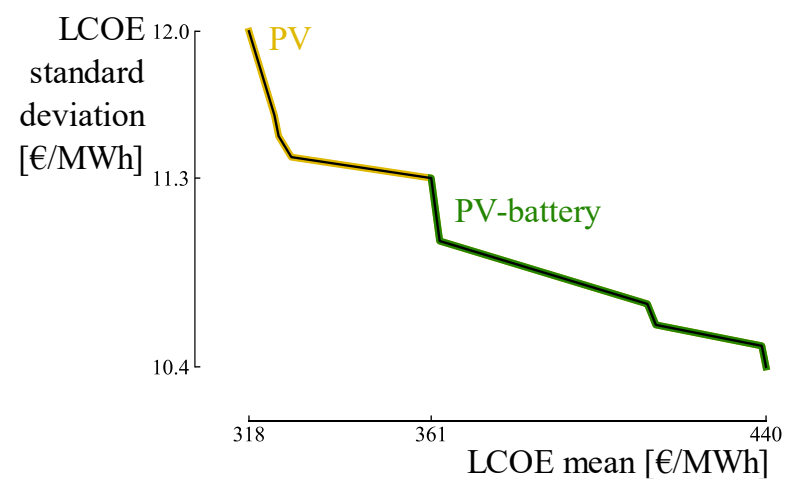

Fig. 2. The Pareto front illustrates the trade-off between minimizing the LCOE mean and minimizing the LCOE standard deviation.

\subsection{Global sensitivity analysis}

Table 2. Characteristics of the three analysed designs.

\begin{tabular}{|c|c|c|c|c|}
\hline & $\begin{array}{c}N_{\mathrm{PV}} \\
{\left[\mathrm{kW}_{\mathrm{p}}\right]}\end{array}$ & $\begin{array}{c}N_{\text {bat }} \\
{[\mathrm{kWh}]}\end{array}$ & $\begin{array}{c}\mu_{\text {max,LCOE }} \\
{[€ / \mathrm{MWh}]}\end{array}$ & $\begin{array}{c}\sigma_{\max , \mathrm{LCOE}} \\
{[€ / \mathrm{MWh}]}\end{array}$ \\
\hline $\begin{array}{c}\mathrm{PV} \\
\text { design }\end{array}$ & 2.2 & 317.6 & 12.0 \\
\hline $\begin{array}{c}\text { PV- } \\
\text { battery } \\
\text { design }\end{array}$ & 3.2 & 1.9 & 361.4 & 11.3 \\
\hline $\begin{array}{c}\text { PV- } \\
\text { battery } \\
\text { design } \\
\text { robust }\end{array}$ & 5.1 & 7.3 & 440.1 & 10.4 \\
\hline
\end{tabular}

Three typical designs are selected out of the set of optimized designs (Table 2): the PV design with the lowest LCOE mean, the PV-battery design with the lowest LCOE mean and the PV-battery robust design (i.e. lowest LCOE standard deviation). The p-boxes of these designs overlap due to the present uncertainty on the system parameters (Fig. 3.). To illustrate for the PVdesign, the true-but-unknown LCOE mean situates between $214 € / \mathrm{MWh}$ and $318 € / \mathrm{MWh}$ and the true-butunknown LCOE standard deviation lies between 0.9 $€ / \mathrm{MWh}$ and $12.0 € / \mathrm{MWh}$. Additionally, it remains inconclusive if these designs are beneficial over full grid-dependency in terms of LCOE. Nevertheless, the PV-design and PV-battery design p-boxes are slightly more beneficial on average than full grid-dependency.

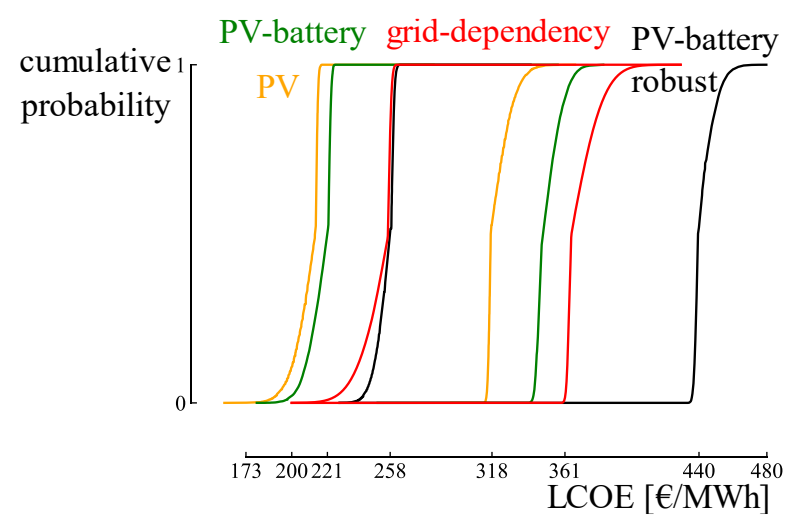

Fig. 3. The probability-boxes of the optimized designs illustrate that due to the present uncertainty on the system parameters, it remains inconclusive if the designs are beneficial over full grid-dependency.

To determine the effect of the input parameter uncertainty on the LCOE standard deviation, the Sobol' indices for the three designs are quantified. Due to the characterization of the LCOE by a p-box, infinite possibilities for the true-but-unknown CDF are present in between the p-box bounds. As each possible CDF corresponds to a deterministic Sobol' index for each input parameter, the Sobol indices related to the p-box are characterized by a precise distribution for each input parameter. As the Sobol' indices indicate the effect of the uncertainty on the LCOE standard deviation, only the stochastic parameters subject to future uncertainty are characterized by a Sobol' index, while the present uncertainty of the stochastic parameters contributes to the variance of the Sobol' indices.

For the PV-design, the distributions of the Sobol' indices illustrate that the future uncertainty on the electricity price and on the solar irradiance are the main contributors to the LCOE standard deviation (Fig. 4.). The future uncertainty on the electricity price corresponds to an average Sobol' index of 0.92, while the future uncertainty on the solar irradiance corresponds to an average Sobol' index of only 0.08 . As both Sobol' index distributions are characterized by a standard deviation of 0.13 , it can be concluded with a $3 \sigma$ confidence level that the future uncertainty on the electricity price dominates the LCOE standard deviation. Hence, improving the determination of the electricity price outcome for every specific scenario is 
presented as a guideline to improve the LCOE robustness of the PV-battery design.

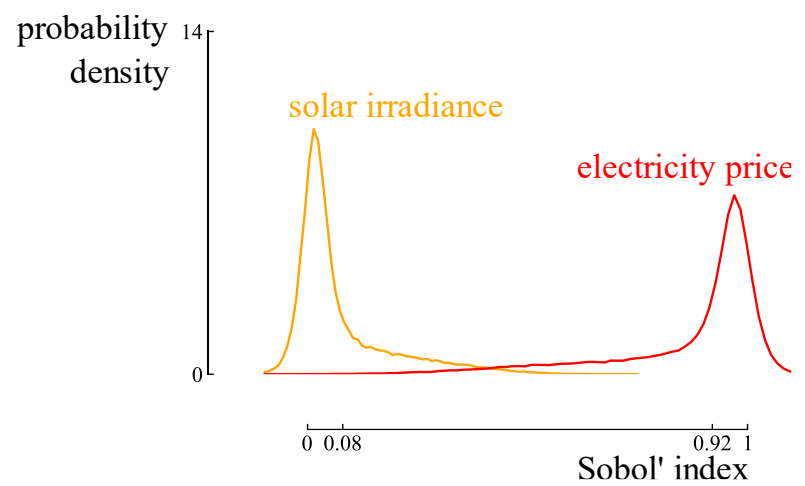

Fig. 4. For the PV-design, the Sobol' indices illustrate the the future uncertainty on the electricity price dominates the LCOE standard deviation.

Similar conclusions can be drawn for the PV-battery design. For this design, the average Sobol' index for the electricity price, solar irradiance and electricity demand correspond to $0.88,0.07$ and 0.05 , respectively (Fig. 5.). Due to the increased SSR of this design as opposed to the PV design, the uncertainty on the electricity demand gains importance in the LCOE standard deviation. Nevertheless, despite the significant standard deviation on the electricity price Sobol' index (0.18), the future uncertainty on the electricity price dominates the LCOE standard deviation with a $3 \sigma$ confidence level.

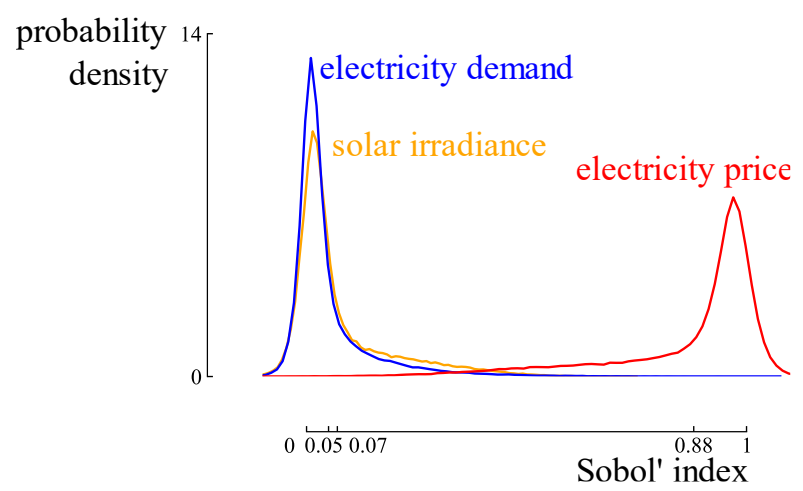

Fig. 5. For the PV-battery design, the Sobol' indices illustrate the the future uncertainty on the electricity price dominates the LCOE standard deviation.

For the PV-battery robust design, the uncertainty on the electricity demand gains significant importance as opposed to the two other designs. The Sobol' index mean and standard deviation of the electricity demand correspond to 0.23 and 0.25 , respectively, while the Sobol' index mean and standard deviation of the electricity price correspond to 0.65 and 0.28 , respectively (Fig. 6.). Hence, due to uncertainty on the Sobol' indices, which is driven by the overall present uncertainty on the system, it remains undetermined which input parameter uncertainty should be addressed to reduce the LCOE standard deviation efficiently. A global sensitivity analysis on these Sobol' index distributions illustrates that the standard deviation on the electricity demand Sobol' index is characterized mainly by the uncertainty on the prediction accuracy of the electricity demand (0.38), the uncertainty on the prediction accuracy of the electricity price $(0.37)$ and the uncertainty on the possible electricity demand scenarios (0.28). The standard deviation of the electricity price Sobol' index is characterized by the uncertainty on the prediction accuracy of the electricity price $(0.70)$, the uncertainty on the prediction accuracy of the electricity demand (0.16) and the uncertainty on the possible electricity demand scenarios (0.15). Conclusively, increasing the level of confidence on the prediction accuracy of the electricity price is the main action to reduce the uncertainty on the Sobol' indices. This action will reveal the most dominant future uncertainty and therefore enables to suggest the appropriate external action to reduce the LCOE standard deviation.

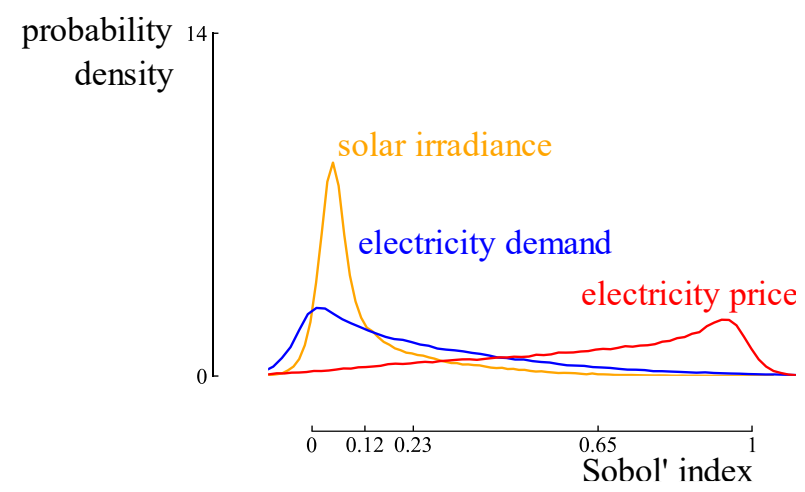

Fig. 6. For the PV-battery robust design, the present uncertainty significantly affects the Sobol' indices.

For all the designs, the present uncertainty on the CAPEX parameters and the interest rate is negligible. Hence, to characterize the most important driver of the LCOE standard deviation, increasing the level of confidence on the CAPEX and interest rate is ineffective. Additionally, both the present and future uncertainty on the ambient temperature have no significant effect on the uncertainty characterization of the LCOE for every optimized design.

\section{Conclusion}

The robust design optimization of a PV-battery system, characterized by a clear distinction between present and future uncertainty, illustrates a trade-off between minimizing the LCOE mean and minimizing the LCOE standard deviation. At the expense of an increase in LCOE mean, the LCOE standard deviation is reduced by subsequently increasing the PV array and battery stack capacity, which subsequently reduces the dependency on grid electricity to comply with the demand.

The p-boxes of an optimized PV-design, PV-battery design and PV-battery robust design illustrate that due to the present uncertainty, it remains inconclusive which designs will outperform full grid-dependency in terms of LCOE in reality. Nevertheless, on average the PVdesign and PV-battery design indicate to be beneficial over full grid-dependency.

To reduce the LCOE standard deviation of the PVdesign and PV-battery design, reducing the future uncertainty related to the grid electricity price is concluded as the most efficient action to improve the 
LCOE robustness. Therefore, improving the determination of the electricity price outcome for every specific scenario is proposed.

For the PV-battery robust design, the present uncertainty becomes significant and should be addressed first. The present uncertainty on the prediction accuracy of the electricity price proves to be the dominant factor and should be reduced by gaining more information on the expected prediction accuracy for each specific scenario. Once this uncertainty is addressed, the most significant guideline to reduce the LCOE standard deviation can be extracted.

For every optimized design, the present uncertainty on the CAPEX parameters and interest rate are negligible in the uncertainty quantification framework and should therefore not be addressed. Moreover, both the present and future uncertainty on the ambient temperature do not influence the LCOE variation of the optimized designs.

Future work aims for the integration of a heat demand, a heat pump and hydrogen-based energy systems.

\section{Acknowledgments}

The first author acknowledges the support of Fonds de la Recherche Scientifique - FNRS [35484777 FRIAB2].

\section{References}

1. International Energy Agency, Tech. Rep., (2018)

2. M. Aneke, M. Wang, Appl. Energy 179, 350-377 (2016)

3. G. Mavromatidis, K. Orehounig, J. Carmeliet, Renew. Sust. Energ. Rev. 88, 258-277 (2018)

4. P. Arun, R. Banerjee, S. Bandyopadhyay, Sol. Energy 83, 1013-1025 (2009)

5. A. Maleki, M.G. Khajeh, M. Ameri, Int. J. Electr. Power Energy Syst. 83, 514-524 (2016)

6. Y. Zheng, B.M. Jenkins, K. Kornbluth, C. Traeholt, Renew Energ. 123, 204-217 (2018)

7. W.F. Holmgren, C.W. Hansen, M.A. Mikofski, Int. J. Open Source Softw. 3, 884 (2018)

8. W. De Soto, S.A. Klein, W.A. Beckman, Sol. Energy 80, 78-88 (2006)

9. S. Blaifi, S. Moulahoum, I. Colak, W. Merrouche, Appl. Energy 169, 888-898 (2016)

10. R. Dufo-López, J.M. Lujano-Rojas, J.L. BernalAgustín, Appl. Energy 115, 242-253 (2014)

11. T. Huld, R. Müller, A. Gambardella, Sol. Energy 86, 1803-1815 (2012)

12. Open Energy Information, Commercial and Residential Hourly Load Profiles for all TMY3 Locations in the United States, Accessed: 4 April 2020

13. M. Montero Carrero, I.R. Sánchez, W. De Paepe, A. Parente, F. Contino, Energies 12, 1-27 (2019)
14. B. Zakeri, S. Syri, Renew. Sust. Energ. Rev. 42, 569-596 (2015)

15. S. Moret, V. Codina Gironès, M. Bierlaire, F. Maréchal, Appl. Energy 202, 597-617 (2017)

16. R. Schöbi, B. Sudret, Reliab. Eng. Syst. Safe. 187 , 129-141 (2019)

17. Elia, Tech. Rep., (2017)

18. Energy Information Administration, Tech. Rep., (2018)

19. D. Devogelaer, D. Gusbin, Federal Planning Bureau, Tech. Rep., (2018)

20. S. M. Wilcox, NREL, Tech. Rep. (2012)

21. N. Lukac, S. Seme, K. Dezan, B. Zalik, G. Stumberger, Energy 107, 854-865 (2018)

22. B. Battke, T. S. Schmidt, D. Grosspietsch, V. H. Hoffmann, Renew. Sust. Energ. Rev. 25, 240-250 (2013)

23. S. Abraham, M. Raisee, G. Ghorbaniasl, F. Contino, C. Lacor, J. Comput. Phys. 332, 461-474 (2017)

24. D. Coppitters, W. De Paepe, F. Contino, Appl. Energy 248, 310-320 (2019)

25. X. Xie, R. Schenkendorf, Processes 7, 509 (2019)

26. K. Verleysen, D. Coppitters, A. Parente, W. De Paepe, F. Contino, Fuel 266, 117049 (2020)

27. W. De Paepe, D. Coppitters, S. Abraham, P. Tsirikoglou, G. Ghorbaniasl, F. Contino, Energy Procedia 158, 5795-5803 (2019)

28. S. Giorgetti, D. Coppitters, F. Contino, W. De Paepe, L. Bricteux, G. Aversano, A. Parente, J. Eng. Gas Turbines Power 142, 011010 (2020) 